\title{
THE PLATELET-TO-LYMPHOCYTE RATIO ON ACUTE COMPLICATION OF DIABETES MELLITUS
}

\author{
Hariogie Putradi, Catur Suci Sutrisnani \\ Department of Clinical Pathology, Faculty of Medicine, Brawijaya University/dr. Saiful Anwar General Hospital Malang, Indonesia. E-mail: \\ hariogie.09@gmail.com
}

\begin{abstract}
Hyperglycemia crisis can occur in Diabetes Mellitus (DM). The uncontrolled complications of DM are Diabetic Ketoacidosis (DKA) and Hyperglycemic Hyperosmolar State (HHS). The inflammatory response potentially occurs in this condition. Platelet-to-Lymphocyte Ratio (PLR) is a new marker of inflammation in which platelet counts tend to increase, while lymphocyte counts tend to decrease due to severe apoptosis. The study purpose was to describe PLR on the acute complication of DM and to know the difference of PLR between DKA and non-DKA (HHS and Mixed). The research was a retrospective study in patients with an acute complication of DM in the dr. Saiful Anwar General Hospital Malang in January 2017-May 2018. The platelet and lymphocyte count were obtained from the Laboratory Information System (LIS). The PLR was calculated by dividing the platelet count by the lymphocyte count. A total of 71 patients were involved in the study, consisting of 21 DKA patients and 50 non-DKA patients. There was a significant difference in platelet count between DKA and non-DKA patients $(p=0.001)$. However, there were no significant differences in lymphocyte count $(p=0.087)$ and $P L R(p=0.762)$ between DKA and non-DKA patients. In DKA, there was a chronic inflammatory process that can affect PLR. As a result, platelet counts tended to increase, while lymphocyte counts tend to decrease due to severe apoptosis. The study showed a significant difference of platelet count between DKA and non-DKA groups, and no significant difference of PLR between DKA and non-DKA groups. It is recommended to conduct further research with a larger sample size.
\end{abstract}

Key words: Diabetes mellitus, diabetic ketoacidosis, hyperglycemia crisis, hyperglycemic hyperosmolar state, the platelet-to-lymphocyte ratio

\section{INTRODUCTION}

Hyperglycemia is a medical condition when blood glucose levels exceed the normal limit. Hyperglycemia is a typical sign of Diabetes Mellitus (DM), although it may also be obtained in other circumstances. Current epidemiological studies show an increased incidence and prevalence of type 2 DM worldwide. The World Health Organization (WHO) estimates that an increase in the number of people with diabetes will be a global health threat. WHO predicts there will be an increase in the number of DM patients in Indonesia from 8.4 million in 2000 to around 21.3 million in 2030. The International Diabetes Federation (IDF) predicts the increase in the number of people with diabetes in Indonesia from 9.1 million in 2014 to 14.1 million in 2035. On the natural history of DM can occur acute and chronic complications. ${ }^{1}$

Acute uncontrolled complications of DM are Diabetic Ketoacidosis (DKA) and Hyperglycemic Hyperosmolar State (HHS), and these conditions have the potential to become a crisis of hyperglycemia. With improved alertness, prevention, and therapeutic guidelines, the mortality rate due to hyperglycemia crisis in the United States in 2009 decreased by 50\% compared to 1980 . Hyperglycemic hyperosmolar state mortality was still higher than DKA and reached 20\%. Hyperglycemic hyperosmolar state incidence is less than 1 case per 1000 people per year. Annual DKA incidents in various reports vary depending on their geographical location. The study reported in the general population in Denmark was 12.9 per 100,000 people. While in Malaysia it is still high at 26.3 per 100 patients peryear. ${ }^{2}$

Diabetic ketoacidosis is a cause of death in $50 \%$ of children and young people with type $1 \mathrm{DM}$ at the age of $<24$ years. Diabetic ketoacidosis can occur in all ages, in a study on 4,807 episodes of DKA, the frequency of DKA in the age of $>70$ years was $14 \%$, age $51-70$ years $23 \%$, age $30-50$ years $27 \%$, age $<30$ years $36 \%$. Another study on $28,770 \mathrm{DM}$ people aged $<20$ years (mean age 14 years), 94\% without episodes of DKA, $5 \%$ with 1 episode of DKA and $1 \%$ had episodes of DKA at least two times. Diabetic 
ketoacidosis is more common in female, people with migrant backgrounds, ages $11-15$ years, with a fatal frequency of $1-5 \% .^{3}$

Diabetic ketoacidosis is characterized by triad of hyperglycemia, anion gap acidosis, and ketonemia. ${ }^{4}$ Criteria for DKA are plasma glucose levels >250 $\mathrm{mg} / \mathrm{dL}$, metabolic acidosis with high anion gap (arterial $\mathrm{pH}<7.3$, serum bicarbonate level $<18 \mathrm{mmol} / \mathrm{L}$, $>10$ anion gap), and the presence of ketones in urine and serum. HHS is characterized by hyperosmolar and dehydration. Hyperglycemic hyperosmolar state criteria are plasma glucose level $>600 \mathrm{mg} / \mathrm{dL}$, increase in effective serum osmolality $>320 \mathrm{mOsm} / \mathrm{kg}$, anion gap <12, no significant acidosis conditions (serum bicarbonate levels $>18 \mathrm{mmol} / \mathrm{L}$ or $\mathrm{pH}>7.3$ ), low ketones levels in urine and serum, and change in mental status. While the mixed conditions (DKA plus $\mathrm{HHS}$ ) are characterized by acidosis $(\mathrm{pH}<7.3$, serum bicarbonate level $<18 \mathrm{mmol} / \mathrm{L}$ ), urine ketone/serum is positive and effective serum osmolality $>320$ $\mathrm{mOsm} / \mathrm{kg}^{5-7}$

Platelet-to-Lymphocyte Ratio (PLR) is a new marker of inflammation and a predictor of various cardiovascular and tumor diseases. Previous studies have shown that DKA is associated with an inflammatory response in the hyperglycemic state. In chronic inflammatory processes, megakaryocytic blood cells proliferate more and more, while lymphocyte counts tend to decrease due to severe apoptosis. As a result, PLR can be affected by severe chronic inflammatory diseases, including the condition of DKA. In this condition, an increase in PLR provides a poor prognosis. Given that the PLR measurement is not expensive and routinely tested in hospitals, PLR can be an ideal predictor of prognosis in patients with DKA. ${ }^{8.9}$

Hyperglycemia can cause excessive oxidation reactions in the tricarboxylic acid cycle, causing an increase in the formation of Reactive Oxygen Species (ROS) resulting in the disruption of mitochondrial function. Fengming et al. showed the presence of mitochondrial dysfunction in platelets, leading to low platelet viability in DM-induced rats and high platelet counts in DM patients. This finding is consistent with the results of Liu et al. study, which showed an increase of platelet counts in DKA patients. $^{8,10}$

The lymphocyte count is also affected by systemic inflammation in the state of DKA. These study from Stentz et al. has reported that leukocytes can produce more ROS in patients with DM. As a result, there is an increase in oxidative damage to lymphocyte DNA in hyperglycemia. These conditions lead to an increase in the proportion of the number of lymphocyte apoptosis, both in DM-induced rats and DM patients. ${ }^{8,10}$

Based on this background, researchers conducted a study describing PLR in patients with acute complications of DM.

This study aimed to determine the description of PLR in acute complications of DM and to know the difference of PLR in DKA and non-DKA (HHS and mixed).

\section{METHODS}

This study was a retrospective study and carried out on all patients with acute complications of DM who were treated in the Dr. Saiful Anwar General Hospital Malang during January 2017-May 2018. Secondary data were obtained from the Laboratory Information System (LIS) data when the patient was first treated at the Dr. Saiful Anwar General Hospital Malang. Platelet-to-lymphocyte ratio was calculated by dividing platelet counts with absolute lymphocyte counts. Then, a descriptive analysis was performed. A total of 71 patients were involved in this study, consisting of 21 DKA patients and 50 non-DKA patients. Patients were classified into DKA group based on the criteria of the American Diabetes Association (ADA): plasma glucose levels $>250 \mathrm{mg} / \mathrm{dL}$, arterial $\mathrm{pH}<7.3$, bicarbonate levels $<18 \mathrm{mmol} / \mathrm{L}$, and the presence of ketones in the urine. While the non-DKA group consisted of HHS and Mixed patients. Hyperglycemic hyperosmolar state criteria were plasma glucose levels $>600$ $\mathrm{mg} / \mathrm{dL}$, bicarbonate levels $>18 \mathrm{mmol} / \mathrm{L}, \mathrm{pH}>7.3$, low ketone levels in urine. While Mixed criteria (DKA plus $\mathrm{HHS}$ ) were $\mathrm{pH}<7.3$, bicarbonate levels $<18 \mathrm{mmol} / \mathrm{L}$, positive urine ketones and effective serum osmolality $>320 \mathrm{mOsm} / \mathrm{kg}$.

Data analysis used the SPSS 23.0 for Windows. Kolmogorov-Smirnov test was used to determine data normality. Unpaired t-test/Mann-Whitney test was used to determine the mean differences between the two groups. In a statistical test, $p<0.05$ was considered a significant value.

The inclusion criteria of this study were all patients with a crisis of hyperglycemia (with glucose levels $>250 \mathrm{mg} / \mathrm{dL}$ accompanied by acute symptoms such as nausea, vomiting, decreased consciousness), there were results of examinations of blood glucose levels, complete blood count, blood gas analysis, and urinalysis from the patient when they first entered the emergency room. While the exclusion criteria for this study were patients with hematologic 
abnormalities (leukemia, aplastic anemia, myelodysplasia syndrome), and patients whose laboratory examination data was incomplete (blood glucose, complete blood count, blood gas analysis, and urinalysis).

The ethichs committe approved the research protocol of the study of Dr. Saiful Anwar General Hospital Malang, no: 400/121/K.3/302/2018.

\section{RESULT AND DISCUSSION}

The sample of this study were patients with acute DM complications, diagnosed clinically, and laboratory-based on ADA criteria. The samples were patients who came to the emergency room of the Dr. Saiful Anwar General Hospital Malang and meets the inclusion and exclusion criteria for clinical and laboratory examinations. Inclusion and exclusion criteria of the sample were determined by history taking, physical examination, complete blood count laboratory examination, blood glucose, blood gas analysis, and urinalysis. The subjects involved during the study period were 71 people who met the inclusion criteria. Of the 71 people divided into two groups, there were 21 DKA groups and 50 non-DKA groups. The general characteristics of patients in this study can be seen in Table 1 .

The results showed that patients with acute DM complications were aged between 7 to 83 years. The number of female patients was more than male. Female patients were 45 (63.4\%) and male were 26 (36.6\%).

The normality test showed abnormal data distribution for age, sex, platelet count, lymphocyte count, PLR, $\mathrm{pH}$, and PDW. While the distribution of data for blood glucose level, $\mathrm{HCO} 3$, and MPV were normal (Table 1).

The results of the comparative test showed significant differences in blood glucose level, platelet counts, $\mathrm{pH}$, and $\mathrm{HCO} 3$ in the DKA and non-DKA groups (Table 1). There were significant differences in platelet counts between DKA and non-DKA patients $(p=0.001)$. However, there were no significant differences in lymphocyte counts $(p=0.087)$ and PLR $(p=0.762)$ between DKA and non-DKA patients.

The age of patients with acute complications of DM in this study was between 7 years to 83 years. DKA is often found in younger patients, while HHS often occurs in patients with older age. However, both DKA and HHS can occur at all ages and even at extreme ages. HHS has been reported in infants aged

Table 1. Characteristics of patients

\begin{tabular}{|c|c|c|c|}
\hline Variable & \multicolumn{2}{|c|}{ Description } & p-value \\
\hline Age & \multicolumn{2}{|c|}{$52.00(7-83)$} & \\
\hline Gender & \multicolumn{2}{|c|}{$\begin{array}{c}\text { Male: } 26(36.6 \%) \\
\text { Female: } 45(63.4 \%)\end{array}$} & \\
\hline & $\begin{array}{c}\text { DKA } \\
(\mathrm{n}=21)\end{array}$ & $\begin{array}{c}\text { Non-DKA } \\
(n=50)\end{array}$ & \\
\hline RBS * & $682.76 \pm 175,53$ & $581,56 \pm 171,17$ & 0.027 ** \\
\hline Thrombocyte & $\begin{array}{c}451.000 \\
(65.000-1.053 .000)\end{array}$ & $\begin{array}{c}285.500 \\
(53.000-1.042 .000)\end{array}$ & 0.001 ** \\
\hline Lymphocyte & $\begin{array}{c}1.763,30 \\
(437,32-6.856,91)\end{array}$ & $\begin{array}{c}1.328,67 \\
(373,88-8.850)\end{array}$ & 0.087 \\
\hline PLR & $\begin{array}{c}234,29 \\
(75.39-2.407,85)\end{array}$ & $\begin{array}{c}237,42 \\
(32.25-885,71)\end{array}$ & 0.762 \\
\hline $\mathrm{pH}$ & $\begin{array}{c}7.09 \\
(6.63-7.31)\end{array}$ & $\begin{array}{c}7.37 \\
(7.06-7.52)\end{array}$ & 0.000 ** \\
\hline $\mathrm{HCO} 3$ * & $6.28 \pm 2.80$ & $17.01 \pm 5.66$ & 0.000 ** \\
\hline PDW & $\begin{array}{c}12.40 \\
(9.40-18.00)\end{array}$ & $\begin{array}{c}12.20 \\
(8.30-25.50)\end{array}$ & 0.364 \\
\hline MPV * & $10.57 \pm 0.91$ & $10.62 \pm 1.42$ & 0.881 \\
\hline
\end{tabular}

Notes: * Normally distributed data presented as mean Standard Deviation (SD)

** $\quad$-value $<0.05$ was considered significant value

RBS = Random Blood Sugar, PLR = Platelet-to-Lymphocyte Ratio, PDW = Platelet Distribution Width,

$\mathrm{MPV}=$ Mean Platelet Volume 
18 months. As many as $20 \%$ of patients with HHS occur under the age of 30 years. Also, DKA has been reported in patients aged over 85 years as much as $4 \%$, about one-third of cases occur in the age range of $55-85$ years. ${ }^{11}$

The number of female patients was more than males, female patients were $45(63.4 \%)$, and males were 26 (36.6\%). According to a multicenter study by Fritsch et al. in Europe, it was found that the incidence of DKA was significantly higher in female patients. ${ }^{12}$ While epidemiological data in the United States show that $50 \%$ of DKA patients were female patients. $^{3}$

The results of the comparative test showed significant differences in blood glucose levels, platelet counts, $\mathrm{pH}$, and $\mathrm{HCO} 3$ in the DKA and non-DKA groups. This result was consistent with the criteria for the diagnosis of DKA and HHS. DKA criteria are plasma glucose levels $>250 \mathrm{mg} / \mathrm{dL}$, metabolic acidosis with a high anion gap (arterial $\mathrm{pH}<7.3$, serum bicarbonate level $<18 \mathrm{mmol} / \mathrm{L}$, anion gap $>10$ ), and the presence of ketones in urine and serum. While the HHS criteria are plasma glucose level $>600 \mathrm{mg} / \mathrm{dL}$, increase in effective serum osmolality $>320 \mathrm{mOsm} / \mathrm{kg}$, anion gap $<12$, no significant acidosis conditions (serum bicarbonate levels $>18 \mathrm{mmol} / \mathrm{L}$ or $\mathrm{pH}>7.3$ ), low ketone level in urine and serum, and changes in mental status. ${ }^{5-7}$

Diagnostic criteria based on ADA Criteria for DKA patient include the presence of blood glucose $>250$ $\mathrm{mg} / \mathrm{dL}$, while for HHS patient presence of blood glucose $>600 \mathrm{mg} / \mathrm{dL}^{5-7}$ In this study, the glucose levels of the DKA patient group were $682.76 \pm 175.53$ (meanSD). This result was following the ADA criteria. Whereas glucose levels in the non-DKA group were $581.56 \pm 171.17$ (mean SD). Included in the non-DKA group in this study were HHS patients and mixed patients (DKA plus HHS). Therefore, the range of glucose levels in this non-DKA group was quite varied, where glucose levels were $>600 \mathrm{mg} / \mathrm{dL}$ for HHS patients and glucose levels $<600 \mathrm{mg} / \mathrm{dL}$ for mixed patients.

Platelet counts increased in the DKA group (median 451,000). In the comparative test, there were significant differences in platelet counts between DKA and non-DKA patients $(p=0.001)$. The condition of hyperglycemia can cause excessive oxidation reactions in the tricarboxylic acid cycle, which causes an increase in the formation of ROS, resulting in the disruption of mitochondrial function. Fengming et al. showed mitochondrial dysfunction in platelets, which led to low platelet viability in animals with DM mice and high platelet counts in DM patients. This finding was consistent with the research of Liu et al. who showed an increase in platelet counts in DKA patients. ${ }^{8,10}$

In this study, there was no significant difference in PLR $(p=0.762)$ between DKA and non-DKA patients. This result could be caused due to limitations in this study. The number of patients who met the inclusion criteria for the DKA group of patients was low (as many as 21 patients) and the total number of samples used in this study was also small (71 patients in total), thus limiting the strength in the analysis of results from the research statistics. Also, Liu et al. studied the PLR cut-off at 267.67 in the DKA patient group. ${ }^{8}$ In patients with high PLR, there is an increased risk of recurrence and death due to DKA. Whereas in Indonesia, there has never been a study of PLR cut-offs in DKA and non-DKA patients so that further research is needed.

In this study, there were also no significant differences in the number of PDW and MPV between DKA and non-DKA patients. In type 1 and 2 diabetes mellitus, increased platelet activity, dysfunction, and morphological changes caused thrombus formation, microvascular embolism, and release of constrictive, oxidative, and mitogenic substances. These changes could be known from PDW and MPV. PDW was affected by an increase in the number and size of platelet pseudopodia. MPV is a marker of function, activity, and size of platelets. The greater amount of MPV, the higher possibility of platelet aggregation. Changes in MPV describe the condition of thrombogenesis. ${ }^{13}$ Mean platelet volume can be affected by glycemic control. Demirtunc et al. reported a significant decrease in MPV after good glycemic control for 3 months in DM patients with $\mathrm{HbAlc}>7 \%{ }^{14}$

\section{CONCLUSION AND SUGGESTION}

The results showed significant differences between platelet counts in DKA and non-DKA patients, and there were no significant differences between PLR in DKA and non-DKA patients. It is recommended to conduct further research with a larger sample size. Also, it is also necessary to research PLR cut-offs in DKA and non-DKA patients.

\section{REFERENCES}

1. Soelistijo SA, Novida H, Rudijanto A, Soewondo P, Suastika K, dkk. Konsensus pengelolaan dan pencegahan diabetes melitus tipe 2 di Indonesia. Jakarta, PB. PERKENI, 2015; 1-82.

2. Maletkovic J, Drexler A. Diabetic ketoacidosis and 
hyperglycemic hyperosmolar state. Endocrinol Metab Clin N Am, 2013; 42: 677-695.

3. Westerberg DP. Diabetic ketoacidosis: Evaluation and treatment. American Family Physician, 2013; 87: 337436.

4. Gotera W, Budiyasa DGA. Penatalaksanaan Ketoasidosis Diabetik (KAD). J Peny Dalam, 2010; 11(2): 126-138.

5. Chou W, Chung MH, Wang HY, Chen JH, Chen WL, et al. Clinical characteristics of hyperglycemic crises in patients without a history of diabetes. J Diabetes Invest, 2014; 5: 657 - 662.

6. Umpierrez GE, Murphy MB, Kitabchi AE. Diabetic ketoacidosis and hyperglycemic hyperosmolar syndrome. Diabetes Spectrum, 2002; 15(1): 28-36.

7. American Diabetes Association. Diagnosis and classification of diabetes mellitus. Diabetes Care, 2018; 27(1): s5 - s10.

8. Liu WY, Lin SG, Wang LR, Fang CC, Lin YQ, et al. Platelet-to-lymphocyte ratio: A novel prognostic factor for prediction of 90-day outcomes in critically ill patients with diabetic ketoacidosis. Medicine, 2016; 95(4): 1-7.

9. Toprak E, Bozkurt M, Çakmak BD, Özçimen EE, Silahl
M, Yumru AE, Çalışkan E. Platelet-to-lymphocyte ratio: A new inflammatory marker for the diagnosis of preterm premature rupture of membranes. Journal of The Turkish German Gynecological Association, 2017; 18(3): 122-126.

10. Stentz FB, Umpierrez GE, Cuervo $R$, Kitabchi AE. Proinflammatory cytokines, markers of cardiovascular risks, oxidative stress, and lipid peroxidation in patients with hyperglycemic crises. Diabetes, 2004; 53(8): 2079-2086.

11. Steenkamp DW, Alexanian SM, McDonnell ME. Adult hyperglycemic crisis: A review and perspective. Curr Diab Rep, 2013; 13: 130-137.

12. Fritsch M, Rosenbauer J, Schober E, Neu A, Placzek K, Holl RW. Predictors of diabetic ketoacidosis in children and adolescents with type 1 diabetes. Experience from a large multicentre database. Pediatr Diabetes, 2011; 12(4 Pt 1): 307-312.

13. Sharma M, Narang S, Nema SK. Study of altered platelet morphology with changes in glycaemic status. Int J Res Med Sci, 2016; 4(3): 757-761.

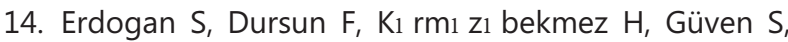
Yildirim UM. Evaluation of erythrocyte and thrombocyte parameters in pediatric patients with diabetes mellitus. J Clin Anal Med, 2017; 8(2): 98-101. 